\title{
A Classic Case of Solid Multiloculated Ameloblastoma of the Mandible
}

\author{
Niharika Prasad ${ }^{1}$ \\ ${ }^{1}$ Assistant Professor, Department of Radiology, Jawaharlal Nehru Medical College, Belagavi, Karnataka, India \\ Corresponding author: Dr. Niharika Prasad, Assistant Professor, Department of Radiology, Jawaharlal Nehru Medical College, \\ Belagavi, Karnataka, India
}

DOI: http://dx.doi.org/10.21276/ijcmsr.2019.4.3.39

How to cite this article: Niharika Prasad. A classic case of solid multiloculated ameloblastoma of the mandible. International Journal of Contemporary Medicine Surgery and Radiology. 2019;4(3):C179-C181.

\section{A B S T R A C T}

Introduction: Ameloblastoma is a benign locally invasive epithelial odontogenic tumor comprising $1 \%$ of all tumors and cysts arising in the jaw. It is usually found in the third and fourth decade in the body ramus region of the mandible. It is associated with a high recurrence rate post excision.

Case report: A young male presented with a few months' history of a painless swelling over jaw. No inflammatory features were present. Routine hematological examination was within normal limits. Computed tomography (CT) plain and contrast showed a well-defined radiolucent expansile lesion involving the right body and ramus of the mandible causing expansion of the body and ramus. Heterogeneously enhancing soft tissue and few bony septations were seen within it. 3- Dimensional image using volume rendering technique (VRT) showed 'soap bubble appearance' of the lesion. Histopathology in this case showed a combination of follicular and plexiform type pattern of ameloblastoma.

Conclusion: In conclusion, ameloblastoma is the most commonly occurring odontogenic tumor in the mandibular body ramus region in the middle age group, however other differential diagnosis such as odontogenic keratocyst, odontogenic myxoma and central giant cell granuloma must be ruled out. CT and MRI have advantages over conventional radiographs as they show better extent and soft tissue involvement.

Keywords: Ameloblastoma, Mandible, Keratocyst, Recurrence

\section{INTRODUCTION}

Mandibular lesions may be odontogenic or non- odontogenic. Odontogenic lesions may be with or without mineralization. As described by H.G.B Robinson, Ameloblastoma is a benign tumor that is usually unicentric, non- functional, anatomically benign and clinically persistent. The World Health Organization (WHO) defines ameloblastoma as a locally invasive polymorphic neoplasia that often has a follicular or plexiform pattern in a fibrous stroma. In $20 \%$ of the cases, it is in the maxilla while in $80 \%$ of the cases, it occurs in the mandible. ${ }^{1}$

The purpose of this report is to describe the imaging findings, differential diagnosis and treatment of Ameloblastomas with detailed discussion of a solid, multiloculated type of Ameloblastoma of the mandible.

\section{CASE REPORT}

A twenty-two-year-old male presented to department of radiodiagnosis, AIIMS, Patna with a painless swelling over right jaw and angle of mouth in the past four months. No history of fever was present. On examination there were no features of inflammation involving skin over the swelling. Lab analysis showed normal blood picture and ESR (erythrocyte sedimentation rate). Plain and contrast computed tomography $(\mathrm{CT})$ images with $3 \mathrm{D}$ reconstruction were obtained.

Computed tomography coronal bone window image showed a large, expansile lesion involving body and ramus of right side of mandible. It was associated with an unerupted tooth (fig 1).

The axial plain CT image of the mandible revealed a very large well-defined radiolucent expansile lesion involving the right body and ramus of the mandible causing expansion

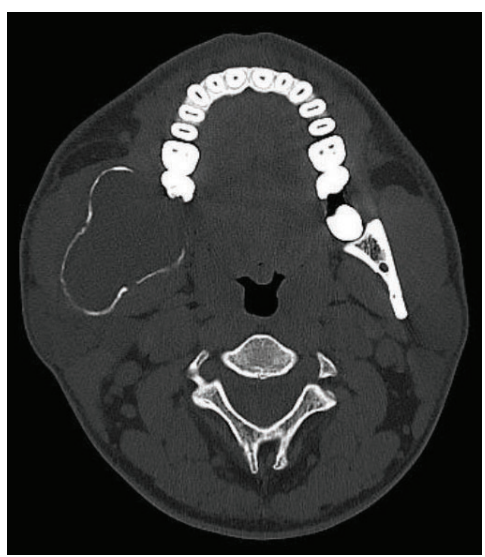

Figure-1: Axial CT bone window image shows a large, expansile lesion involving body and ramus of right hemimandible. An unerupted tooth is also seen on the right side. 


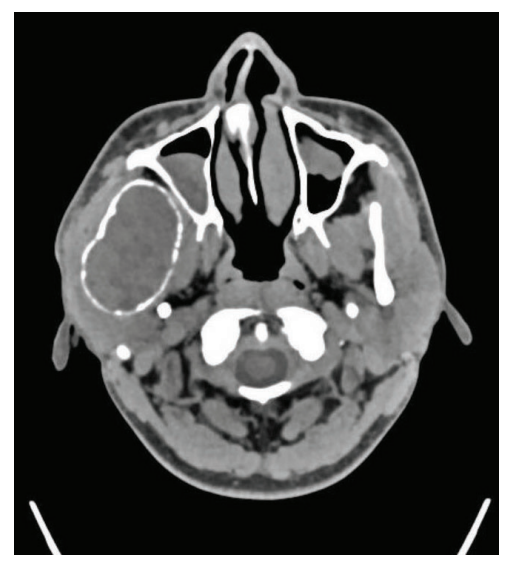

Figure-2: Axial plain CT image of the mandible shows soft tissue density within the lesion. No calcifications were seen within it. Few foci of break in continuity of osseous margin are seen, however there is no perilesional extension of soft tissue.

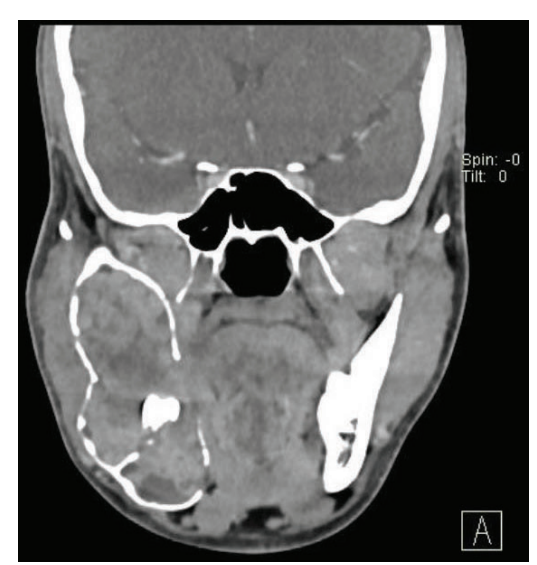

Figure-3: Coronal post contrast CT image shows heterogeneous enhancement within the lesion. There were no hemorrhagic fluid-fluid levels within the lesion.

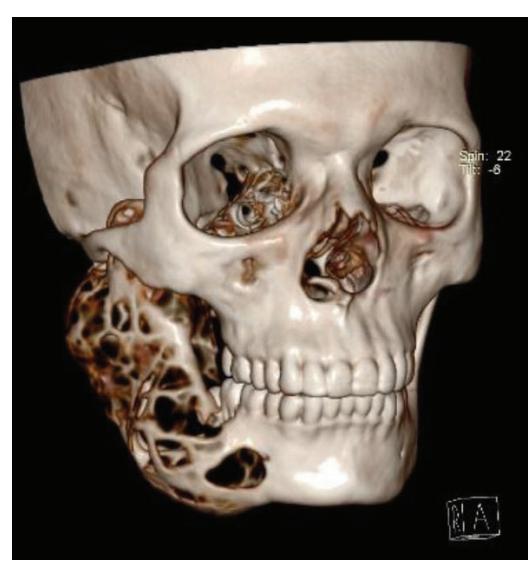

Figure-4: 3-D image using volume rendering technique (VRT) shows the expansile multiloculated lesion with classical honeycomb or "soap- bubble appearance".

of the body and ramus. Soft tissue density and few bony septations were seen within it. Few foci of break in continuity of osseous margin was seen, however there was no perilesional extension of soft tissue.

Fig 2 shows the axial and coronal post contrast CT images show heterogeneous enhancement within the lesion. Fig 3 and fig 4 shows 3- Dimensional image using volume rendering technique (VRT) showed the expansile multiloculated lesion with a classical "soap- bubble appearance".

\section{DISCUSSION}

Ameloblastomas constitute approximately 1\% of all tumors of the mandible and usually present in 3rd and 4th decades of life as a painless swelling. ${ }^{1}$ They don't have any gender predilection. Most Ameloblastomas are benign. They are odontogenic tumors composed of epithelial elements, histologically seen as follicular (more common) or plexiform patterns. Histopathology in this case showed a combination of follicular and plexiform type pattern. Basaloid, granular and desmoplastic types are other less common variants. However, the biological behavior cannot be predicted by their histology.

Most common location ( 80\%) for this lesion is body ramus region of the mandible while rest of them arise in the maxilla, in the maxillary tuberosity. ${ }^{1}$ There are four radiographic patterns described in literature- a) Unicystic type, b) Spider-web pattern, c) Soap bubble/multilocular pattern and d) Honeycomb or solid pattern. They have a tendency for extensive root resorption. ${ }^{2}$ Tooth displacement and destruction of inferior alveolar canal can be other findings. On imaging, Ameloblastomas usually have well defined, frequently corticated and

occasionally scalloped margins. ${ }^{2}$ The investigations include Panoramic radiographs, computed tomography, magnetic resonance imaging and biopsy. A review article ${ }^{6}$ based on retrospective study of a series of panoramic radiographs classified radiolucent lesions into those with well-defined and ill-defined borders. Lesions which show well-defined borders can include radicular cyst, residual cyst, dentigerous cyst, keratocyst, ameloblastoma, eosinophilic granuloma and simple bone cyst. Lesions with ill-defined borders include osteomyelitis, osteoradionecrosis, osteoblastoma, squamous cell carcinoma and metastasis. Pseudo lesions can also occur in the mandible like Stafne cyst which is a pseudocyst arising from bone remodeling caused by the adjacent submandibular gland. Radiological features such as large lesion size, bone scalloping, relationship to an impacted tooth or the mandibular canal, tooth resorption, as well as ill-defined lesion borders, require further radiological work-up. A CT is usually helpful for determining the margins of the lesion, its contents, and its extension into soft tissues. ${ }^{2}$ Though most radiolucent lesions with well-defined sclerotic borders are thought to be benign, MRI may reveal clinically unsuspected malignant disease. Such accurate assessment is the drawback of panoramic radiographs. As an exception to previously mentioned radiographic patterns, the desmoplastic variant of ameloblastoma often manifests in maxilla as a mixed radiolucent and sclerotic lesion, resembling a benign fibro osseous lesion. ${ }^{3}$

The lesion may resemble an odontogenic keratocyst and sometimes the differentiation may be possible only on histopathological correlation. ${ }^{4}$ There are, however, a few points which may aid in the differentiation radiologically. According to literature odontogenic keratocysts have lower CT density with higher heterogeneity than Ameloblastomas. ${ }^{5}$ One of 
the studies showed a significant difference regarding bony expansion, impacted teeth and high-density areas between the two. ${ }^{5}$ According to this study, the presence of highdensity areas is the most useful feature in the differential diagnosis of ameloblastomas and keratocystic odontogenic tumors.

The treatment of ameloblastoma is surgery with wide resection to high recurrence rate especially of solid/muticystic variety. Recurrence rate after excision has been reported in up to $33 \%$ of reported cases. ${ }^{1}$ The recurrence rate after curettage is much higher (90-100\%). ${ }^{3}$ Radiotherapy can be considered for patients with positive margins who are not amenable to re- excision. ${ }^{3} \mathrm{~A}$ margin of $1.5-2 \mathrm{~cm}$ beyond the radiological limit to ensure all microcysts are removed.

Final diagnosis was made as Multiloculated type of ameloblastoma of the mandible.

\section{CONCLUSION}

Although very often the diagnosis of ameloblastoma is made on basis of radiographic features, one should never rely on it alone. In conclusion, all such lesions should be biopsied, and an accurate histologic diagnosis should be obtained before definitive treatment is started. ${ }^{2}$

\section{REFERENCES}

1. Weissman JL, Snyderman CH, Yousem SA, Curtin HD. Ameloblastoma of the maxilla: CT and MR appearance. AJNR Am J Neuroradiol. 1993;14(1):223-6.

2. More C, Tailor M, Patel HJ, Asrani M, Thakkar K, Adalja C. Radiographic analysis of ameloblastoma: a retrospective study. Indian J Dent Res. 2012;23(5):698.

3. Bachmann AM, Linfesty RL. Ameloblastoma, solid/ multicystic type. Head Neck Pathol. 2009; 3(4):307-9.

4. Bajpai M, Agarwal D, Bhalla A, Kumar M, Garg R, Kumar M.Multilocular unicystic ameloblastoma of mandible. Case Rep Dent. 2013;835892.

5. Ariji $\mathrm{Y}$ et al. Imaging features contributing to the diagnosis of ameloblastomas and keratocystic odontogenic tumours: logistic regression analysis. Dentomaxillofac Radiol. 2011; 40(3):133-40.

6. Avril L, Lombardi T, Ailianou A, et al. Radiolucent lesions of the mandible: a pattern-based approach to diagnosis. Insights Imaging. 2014;5(1):85-101.

7. Meyer KA, Bancroft LW, Dietrich TJ, Kransdorf MJ, Peterson JJ.Imaging characteristics of benign, malignant, and infectious jaw lesions: a pictorial review. American Journal of Roentgenology. 2011;197(3): W412-21.

8. Singh M, Shah A, Bhattacharya A, Raman R, Ranganatha N, Prakash P. Treatment algorithm for ameloblastoma. Case reports in dentistry. 2014;2014.

9. Devenney-Cakir B, Subramaniam RM, Reddy SM, Imsande H, Gohel A, Sakai O. Cystic and cysticappearing lesions of the mandible. American Journal of Roentgenology. 2011;196(6):WS66-77.

10. Dandriyal R, Gupta A, Pant S, Baweja HH. Surgical management of ameloblastoma: Conservative or radical approach. Natl J Maxillofac Surg 2011; 2(3):22-7.

Source of Support: Nil; Conflict of Interest: None

Submitted: 25-07-2019; Accepted: 22-08-2019; Published online: 15-09-2019 